\title{
MOLECULAR DYNAMICS SIMULATION OF ADHESION FORCES IN A DIPALMITOYLPHOSPHATIDYLCHOLINE MEMBRANE
}

\author{
JUN YIN and YA-PU ZHAO* \\ State Key Laboratory of Nonlinear Mechanics (LNM), \\ Institute of Mechanics, Chinese Academy of Sciences, \\ Beijing 100080, People's Republic of China \\ *yzhao@imech.ac.cn
}

Received 19 December 2006

\begin{abstract}
Adhesion forces of Dipalmitoylphosphatidylcholine (DPPC) membrane in the gel phase are investigated by molecular dynamics (MD) simulation. In the simulations, individual DPPC molecules are pulled out of DPPC membranes with different rates and we get the maximum adhesion forces of DPPC membrane. We find that the maximum adhesion forces increase with pull rate, from about 400 to $700 \mathrm{pN}$ when pull rates are from 0.001 to $0.03 \mathrm{~nm} / \mathrm{ps}$. We analyze the relationship between pull rate and adhesion forces of different origins using Brownian dynamics and notice that viscosity of solvent plays an important role in adhesion forces. Then we simulate the motion of a single DPPC molecule in solvent and it elucidates that the maximum drag force is almost linear with respect to the pull rate. We use Stokes' relation to describe the motion of a single DPPC molecule and deduce the effective length of a DPPC molecule. Conformational analyses indicate that the free energy variation of a DPPC molecule inside and outside of the DPPC membrane is an essential part of adhesion energy.
\end{abstract}

Keywords: Dipalmitoylphosphatidylcholine (DPPC); molecular dynamics; adhesion forces; Brownian dynamics.

\section{Introduction}

Knowledge of the structure and dynamics of biomembrane has attracted considerable interest during the past decades, because of its fundamental importance in biological systems. Biological membranes enclose every cell and numerous intracellular organelles. The main biological functions of membranes involve various life processes, such as receiving and transducing signals between cells, separation of intracellular compartments, and control of interactions between the cell and its environment. The predominant interest in phospholipid bilayers arises because they are one of the major, if not the major, structural elements of biological membranes. ${ }^{1}$ DPPC is one of the best studied lipids, both by experiment and simulation. $^{2-5}$

In our work, we assumed that the intensity of adhesion forces of biomembranes is equal to the force applied to drive lipids away from their equilibrium position. The adhesion forces of biomembrane are of

\footnotetext{
${ }^{*}$ Corresponding author.
} 
general interest to cell binding, fusion, and protein insertion, especially important for the anchoring of proteins to a membrane. ${ }^{3}$ Some molecular devices such as molecular motors mainly work on biomembrane; so adhesion forces of biomembrane are essential for the stability of these devices.

In our simulations, individual DPPC molecules are pulled out from DPPC membranes and we get the maximum adhesion forces of DPPC membrane in each process, and the maximum adhesion forces with different pull rates are found. Then we simulate the motion of a single DPPC molecule in solvent and it elucidates that the maximum drag forces are almost linear with respect to different pull rates.

\section{Simulation Methods}

In our simulations, we apply the force field and simulation parameters that have been invented by Berger et al. ${ }^{4}$ for a DPPC membrane. The DPPC membrane is composed of 64 lipids, and there are about $5 \mathrm{~nm}$ length water layers on both sides of the DPPC membrane. We use single point charge (SPC) water model in the simulations, because it is proved that the effective free energy of the SPC model is close to the experimental value than that of the extended single point charge (SPC/E) model. ${ }^{6}$

In all simulations, a time step of $1 \mathrm{fs}$ is used. The cutoff of Lennard--Jones (LJ) interaction is $1.5 \mathrm{~nm}$, while the cutoff of electrostatic interaction is $2.0 \mathrm{~nm}$. All simulations are performed with NPT ensemble and temperature coupling at $300 \mathrm{~K}$, where the coupling constant $\tau=0.1 \mathrm{ps}$. Since the transition temperature of DPPC for the transition between the gel and liquid crystalline phases of DPPC membrane is $315 \mathrm{~K},{ }^{7}$ in all the simulations, the DPPC membranes are in gel phase. Pressure coupling is used with a coupling constant $\tau=1.0 \mathrm{ps}$, at 1 bar in all three directions. All simulations include two parts, pulling a DPPC molecule out from a DPPC membrane and the motion of a single DPPC molecule in solvent. In all pulling simulations, the spring constant of virtual spring is $1560 \mathrm{KJ} / \mathrm{mol} \cdot \mathrm{nm}^{2}{ }^{8}$

At each pull rate, we perform five independent simulations. There are two data-averaging methods, ensemble-averaged method, and individually averaged method, respectively. In ensemble-averaged method, we average the profile first, and then determine the maximum adhesion force or the maximum drag force, while in individually averaged method, we determine the maximum adhesion force or the maximum drag force first, and performed average afterward.

\section{Results}

In order to study the relationship between the pull rate and adhesion force, we simulate at seven different pull rates: $0.001,0.0015,0.002,0.005,0.01$, 0.02 , and $0.03 \mathrm{~nm} / \mathrm{ps}$, respectively. Figure 1 shows the profiles of adhesion force with pulling distance at pull rate $0.002 \mathrm{~nm} / \mathrm{ps}$. We carry out the Fast Fourier Transform (FFT) to filter out fast frequencies arising from random thermal motion. In Fig. 1, we find that the maximum adhesion force is reached when a DPPC molecule is pulled out almost $2 \mathrm{~nm}$, and the maximum adhesion force in the individual profile $(\sim 500 \mathrm{pN})$ is larger than that in the averaged profile $(\sim 300 \mathrm{pN})$. The maximum adhesion forces in our simulations are an order larger than rupture force $(\sim 70 \mathrm{pN})$ of the lipid-membrane complex in experiments. ${ }^{9}$ One primary reason would be that the pull rates in our simulations are much faster than those in experiments.

Figure 2(A) shows the relationship between the pull rate and the maximum adhesion forces, and we find that the maximum adhesion forces increase with pull rate. In the slow pull rate region $(<0.002 \mathrm{~nm} / \mathrm{ps})$, the maximum adhesion forces increase linearly with pull rate, but it becomes nonlinear in high pull rate region $(>0.002 \mathrm{~nm} / \mathrm{ps})$.

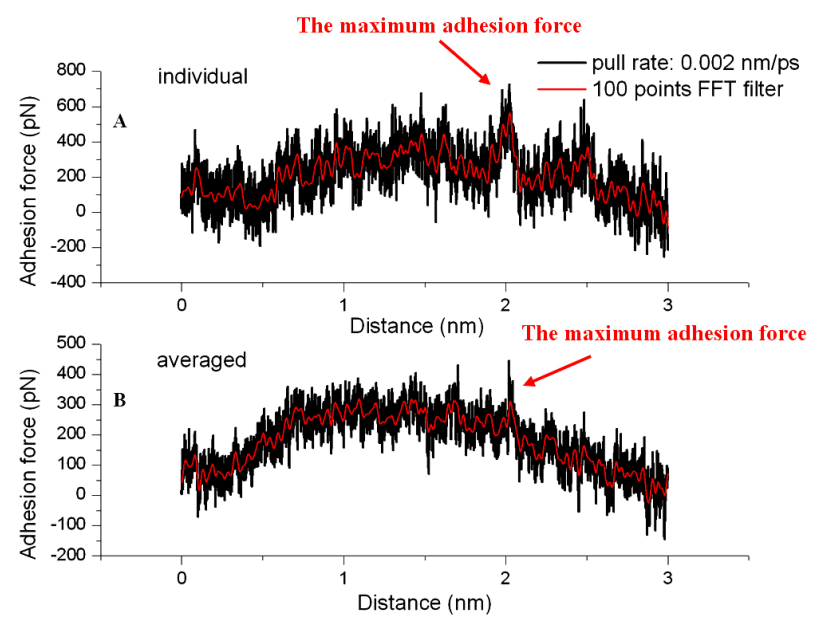

Fig. 1. (A) An individual profile of adhesion force; (B) An averaged profile over five simulation times. 


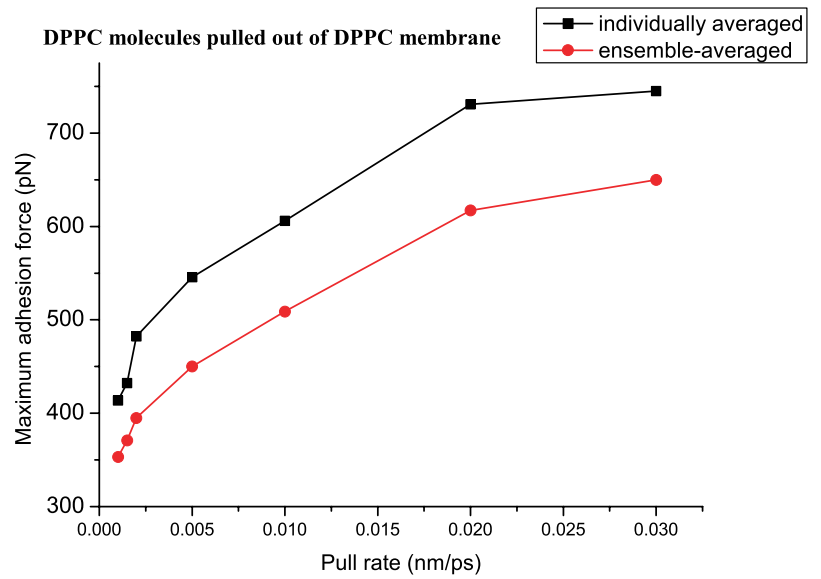

(A)

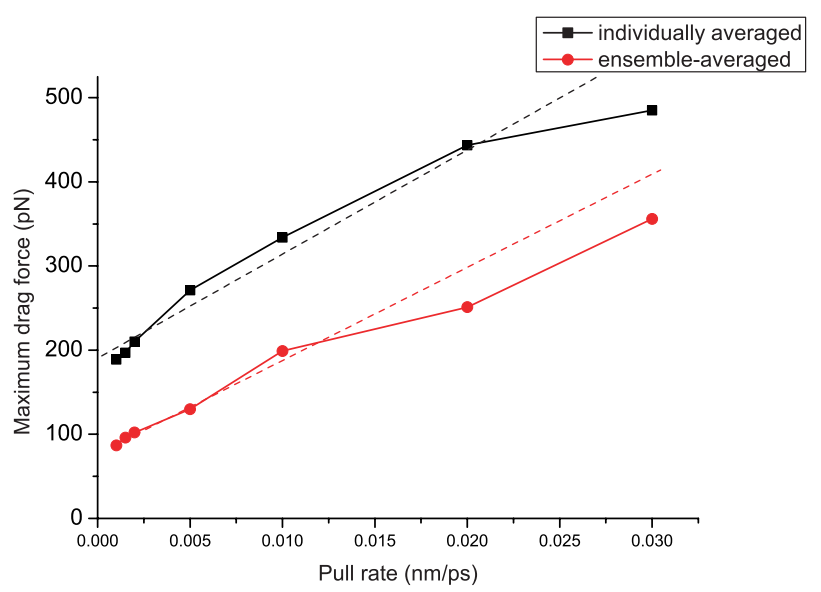

(B)

Fig. 2. (A) The maximum adhesion forces; (B) The maximum dragging forces. The dashed lines are the leastsquares linear fit of two forces profiles.

These results are in accordance with Arrink et al.'s simulations of DPPC membrane in liquid crystalline phase. $^{3}$

Obviously, drag force of solvent is a significant part of adhesion force. In Fig. 2(B), we notice that the maximum drag forces of a single DPPC molecule in solvent, both individually averaged, and ensemble-averaged increase linearly with pull rates. It is reasonable to use Stokes' relation to describe this phenomenon. The drag force of solvent is given by

$$
F=-\alpha v=-a \eta v,
$$

where $\alpha$ is the drag coefficient, $v$ is pull rate, $a$ is the effective length of a DPPC molecule, and $\eta$ is the dynamic viscosity coefficient of solvent. In water solvent, $\eta \approx 1.14 \times 10^{-3} \mathrm{~Pa} \cdot \mathrm{s}$, and we assume drag force $F$ is almost equal to the maximum drag force in Fig. 2(B). From Eq. (1), we get the effective length of a DPPC molecule $a=8.38 \mathrm{~nm}$.

\section{Discussions}

\subsection{The motion of a single DPPC molecule}

Because of the universal existence of molecular thermal motion, we use Brownian dynamics to describe the random motion in the system. For the motion of a single DPPC molecule in the solvent, the Langevin equation is

$$
m \frac{d v}{d t}=F(t)+R(t)-\alpha v=F(t)+R(t)-\gamma p,
$$

where $F(t)$ are external forces, $R(t)$ are random forces of molecule in system, and $-\alpha v$ is the drag force, while $\gamma=\alpha / m$, and $p=m v$ is the momentum. We get the solution of Eq. (2),

$$
p(t)=\mathrm{e}^{-\gamma t}\left[p(0)+\int_{0}^{t}[F(\xi)+R(\xi)] \mathrm{e}^{\gamma t} d \xi\right] .
$$

There is a decay time $1 / \gamma$ in Eq. (3), which is a timescale for random motion. In Eq. (1), $1 / \gamma=$ $m / \alpha=m / a \eta$. In our system, $a \approx 8.38 \mathrm{~nm}, \eta \approx 1.14 \times$ $10^{-3} \mathrm{~Pa} \cdot \mathrm{s}$, and for DPPC molecule, $m \approx 650 \mathrm{~g} / \mathrm{mol}$ (without hydrogen atoms); so we evaluate the decay time $1 / \gamma \sim 10^{-13} \mathrm{~s}$. In our simulations, the time step $d t=10^{-15} \mathrm{~s} \ll 1 / \gamma$; therefore, the random thermal force would not decay remarkably in every step. When we calculate drag force, we should include the random thermal force of molecules.

Considering conservation of energy when pulling a single DPPC molecule in solvent, we could use Brownian dynamics again. Because in the direction of pulling $Z$, we transform Langevin equation, Eq. (2) to

$$
F(z)=m v \frac{d v}{d z}+\alpha v-R(z) .
$$

Integrating Eq. (4) on direction $Z$, we get

$$
\int_{0}^{l} F(z) d z=\frac{1}{2} m v^{2}+\int_{l}^{0} \alpha v d z-\int_{l}^{0} R(z) d z .
$$

The term on the left of Eq. (5) is the pulling work of external force, while on the right, the first term is the kinetic energy of DPPC molecule, the second 
term is the work of solvent viscosity, and the third term is the energy of thermal motion of system.

Now we discuss about three terms on the right of Eq. (5). For the kinetic energy term, when $v=$ $0.03 \mathrm{~nm} / \mathrm{ps}$ (the fastest pull rate in all simulations),

$\frac{1}{2} m v^{2} \sim 1 \mathrm{pN} \cdot \mathrm{nm} \ll \int_{l}^{0} F(z) d z \sim 10^{3} \mathrm{pN} \cdot \mathrm{nm}$.

For the work of solvent viscosity, according to Stokes' relation, we have

$$
\int_{l}^{0} \alpha v d z=a \eta v L
$$

where $L=3 \mathrm{~nm}$ is the pull length in simulations. When $a \approx 8.38 \mathrm{~nm}$ and the dynamic viscosity coefficient of water $\eta \approx 1.14 \times 10^{-3} \mathrm{~Pa} \cdot \mathrm{s}$, we get $\int_{l}^{0} \alpha v d z \sim$ $1000 \mathrm{pN} \cdot \mathrm{nm}$, which is of the same order of pulling work of external force. In principle, the magnitude of thermal motion energy depends on temperature only; so for the third term, we have $\int_{l}^{0} R(z) d z \sim K_{B} T$, where $K_{B}$ is Boltzmann constant and $T=300 \mathrm{~K}$ is the absolute temperature. So $K_{B} T=4.14 \mathrm{pN} \cdot \mathrm{nm} \ll$ $10^{3} \mathrm{pN} \cdot \mathrm{nm}$. Therefore, work of solvent viscosity plays a dominating role in pulling work of external force, while the kinetic energy and thermal motion energy can be neglected.

\subsection{Adhesion forces of DPPC membrane}

As to the adhesion forces of DPPC membrane, Marrink et $a .^{3}{ }^{3}$ suggested that the total adhesion force can be expressed as an interplay between forces of three different origins, i.e.

$$
F_{\text {adh }}=F_{\text {thd }}+F_{\text {fr }}+F_{\text {rand }},
$$

where $F_{\text {thd }}$ is the thermodynamic component, which arises from the free energy barrier that exits along the pulling path, $F_{\mathrm{fr}}$ is the friction component, which originates from nonrandom collisions with neighboring molecules, and $F_{\text {rand }}$ is the random component, which arises from the random thermal motion of the system.

Because of the thermodynamic component $F_{\text {thd }}$, we add the term of free energy variation of the pulled DPPC molecule to Eq. (5) and get

$\int_{l}^{0} F(z) d z=\frac{1}{2} m v^{2}+\int_{l}^{0} \alpha v d z-\int_{l}^{0} R(z) d z+\Delta G$, to describe the pulling of a DPPC molecule out from the DPPC membrane. The free energy variation of a pulled DPPC molecule is

$$
\Delta G \approx A(z)\left(\gamma_{\mathrm{DPPC}-\text { water }}-\gamma_{\mathrm{DPPC}-\mathrm{DPPC}}\right),
$$

where $A(z)$ is the new interface area formed between the pulled DPPC molecule and solvent, $\gamma_{\mathrm{DPPC}-\text { water }}$ is the interface energy of DPPC molecule and solvent (water in our simulations), and $\gamma_{\mathrm{DPPC}-\mathrm{DPPC}}$ is the interface energy of DPPC molecules. Since the main part of DPPC molecule is the two hydrophobic hydrocarbon chains, we get $\gamma_{\text {DPPC-DPPC }}<$ $\gamma_{\text {DPPC-water. }}$ In other words, $\Delta G>0$.

When a DPPC molecule is pulled from the DPPC membrane into water, because of hydrophobicity of DPPC hydrocarbon chains, a folded conformation is energetically more favorable because it minimizes the area of water-exposed methyl groups. However, the friction force $F_{\mathrm{fr}}$ makes a stretched conformation more favorable. Since $F_{\text {fr }}$ increases with pull rate, the pulled DPPC molecule attains more stretched conformation at high pull rate (Fig. 3). As the pulled DPPC molecule extends, the new interface area $A(z)$ increases, which leads to the increase of $\Delta G$. From Eq. (9), the increase of $\Delta G$ with pull rate is a reason for the increase of adhesion force of DPPC membrane.
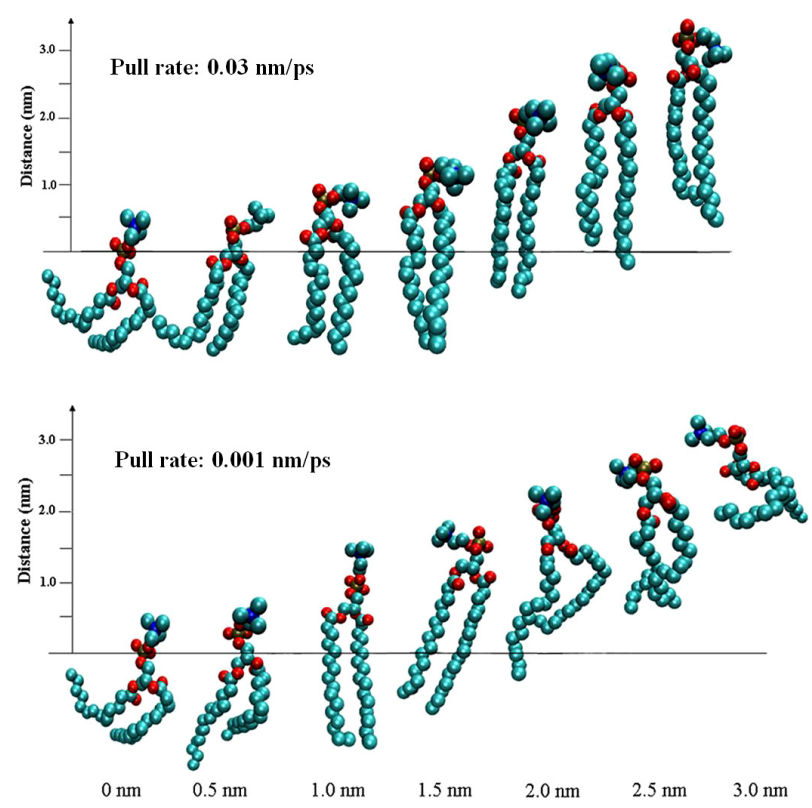

Fig. 3. Snapshots of typical conformations when pulled out from DPPC membrane at different rates: $0.03 \mathrm{~nm} / \mathrm{ps}$ and $0.001 \mathrm{~nm} / \mathrm{ps}$, respectively. 


\section{Conlusions}

We provide the simulations of DPPC membrane adhesion forces and the motion of a single DPPC molecule in solvent. First, we estimate the effective length of a DPPC molecule using Stokes' relation. And then we find that both the maximum adhesion forces and the maximum drag forces increase with pull rate. We analyze the forces from different origins using Brownian dynamics and notice that viscosity of solvent plays an important role. Besides, free energy variation of a DPPC molecule inside and outside the DPPC membrane also affects the maximum adhesion force of a DPPC membrane.

\section{Acknowledgments}

This work was supported by the National Basic Research Program of China (973 Program, Grant No. 2007CB310504), Distinguished Young Scholar Fund of National Natural Science Foundation of China (NSFC, Grant No. 10225209) and key project from Chinese Academy of Sciences (Grant No. KJCX-SW-L2).

\section{References}

1. G. Cevc and D. Marsh, Phospholipid Bilayers (John Wiley and Sons, Inc., New York, 1987).

2. Z. V. Leonenko, E. Finot, H. Ma, T. E. S. Dahms and D. T. Cramb, Biophys. J. 86 (2004) 3783.

3. S. J. Marrink, O. Berger, P. Tieleman and F. Jahnig, Biophys. J. 74 (1998) 931.

4. O. Berger, O. Edholm and F. Jahnig, Biophys. J. 72 (1997) 2002.

5. J. Landin, I. Pascher and D. Cremer, J. Phys. Chem. 99 (1995) 4471.

6. D. P. Tieleman and H. J. C. Berendsen, J. Chem. Phys. 105 (1996) 4871.

7. M. J. Janiak, D. M. Small and G. G. Shipley, Biochemistry 15 (1976) 4575.

8. H. Grubmuller, B. Heymann and P. Tavan, Science 271 (1996) 997.

9. D. Leckband, W. Muller, F. Schmitt and H. Ringsdorf, Biophys. J. 69 (1995) 1162. 